\title{
Coronary angiography finding of exercise tread mill test positive patients in a tertiary care center
}

\author{
Amit Shrestha, Sanjeev Thapa, Sheelendra Shakya, Ravi Shahi, Chandra Mani Paudel, Surya Raj Pathak
}

Department of Cardiology, Manmohan Cardio Vascular Thoracic and Transplant Centre, Institute of Medicine Tribhuwan University Teaching Hospital, Kathmandu, Nepal

Corresponding Author: Amit Shrestha

Department of Cardiology,

Manmohan Cardio Vascular Thoracic and Transplant Centre,

Institute of Medicine, Tribhuwan University Teaching Hospital, Kathmandu, Nepal,

Email Address: amitshrestha32@hotmail.com

\begin{abstract}
Background and Aims: The use of coronary angiography in diagnosing coronary artery disease is limited by its invasive property. In the other hand correct interpretation of tread mill test data and its use as a key diagnostic modality also has been a problem. The study was thus aimed to see the diagnostic accuracy of treadmill test to rule out coronary artery disease.

Methods: : We included all the patients who had positive tread mill stress test and underwent coronary angiography and were subsequently analyzed for presence of coronary artery disease.

Results: A total of 303 patients were included with 119 males and 184 females with mean age of $53.6 \pm 10.5$ yrs and $51.7 \pm 8.6$ yrs respectively. Normal coronaries was seen in $114(54.0 \%)$, borderline lesion in $29(13.7 \%)$ and significant lesion in $68(32.2 \%)$ with 48(22.7\%) having single vessel disease, $29(13.7 \%)$ double vessel disease and 14(6.6\%) triple vessel disease. Coronary artery diseases was highest among diabetics (57.7\%, OR 1.72 (95\% CI: 0.92 to 3.20), p value-0.08).Similarly the risk of coronary artery disease was significantly highest among patient with $\geq 2$ risk factor (OR: $8.10,95 \%$ CI: 4.96 to $13.24, \mathrm{P}<0.0001)$. Gender distribution showed that coronary artery disease was significantly higher in males than females (53\% vs $35 \%$ respectively, OR: $2.08,95 \%$ CI: 1.30 to 3.32 , p value- 0.002 ).

Conclusion: The value of tread mill test to predict coronary artery disease is highest in patients with two or more risk factor especially in those with diabetes with significance increased among males.
\end{abstract}

Keywords : Coronary angiography; Coronary artery disease ; Tread mill stress test.

\section{Introduction}

The diagnostic rate of coronary artery disease $(\mathrm{CAD})$ has been dramatically increasing with the development of interventional technique, which makes coronary angiography (CAG) the "gold standard" tool for CAD diagnosis. ${ }^{1}$ However, limited by its invasive property exercise treadmill test is still used as an economic and simple method to screen and assist in diagnosis of patients with known or suspected CAD. ${ }^{1}$ The study is thus retrospectively looking at coronary angiographic findings of those with tread mill test positive status .

Another factor is evaluation of ECG changes, specifically ST segment depression during or after exercise. However, with chances of false positive and negative tests, especially in patients with atypical or no angina pectoris is one of the problem of test being not highly specific. ${ }^{2}$ Thus, use of the test in specific population to increase the pretest diagnostic accuracy is the main rationale of this study.

In the study we aim to look at the overall prevalence of CAD in exercise tread mill test positive patients and the parameters that affect its diagnostic accuracy.

\section{Methods}

\section{Study Design}

This was a quantitative cross sectional retrospective study Place and duration of study

The study was done in the department of cardiology, Manmohan Cardiothoracic Vascular and Transplant Center, Institute of medicine, Maharajgunj, Kathmandu in a study period of approximately 1 and $1 / 2$ yrs (Oct 2015 to Dec 2016)

\section{Ethical approval and patient consent}

The study was started only after ethical clearance for the study was taken from Institutional review board, Institute of medicine. Informed and written consent were taken from all the patients in the study group.

\section{Inclusion and Exclusion Criteria}

Inclusion Criteria

- All patients with exercise tread mill test positive (based on ACC/AHA 2002 guidelines)3 undergoing coronary 
angiography. The following results will be interpreted as positive

\section{During Excercise}

- ST segment depression: ECG portion of the exercise test will be considered abnormal (ie, "positive" for ischemia) when there is $\geq 1 \mathrm{~mm}$ horizontal or downsloping ST segment depression in one or more leads that persists at 80 milliseconds after the $\mathrm{J}$ point especially in Leads V4, V5, and V6 or V5 alone. ${ }^{4}$

- $\quad \mathrm{ST}$ segment elevation : $\geq 1.0 \mathrm{~mm}$ ST segment elevation in leads without pre-existing $\mathrm{Q}$ waves

- ST segment elevation in aVR

- Exertional hypotension: Fall in Systolic Blood Pressure from pre-exercise value as exercise intensity increases

- Exercise capacity: Inability to achieve 3 MET workload

- Exercise-induced angina, particularly that which is exerciselimiting or occurs at a low workload

- Heart rate response to exercise or chronotropic incompetence (failure to achieve 85 percent of the age-predicted maximum heart rate in the absence of medications known to slow or blunt the heart rate response to exercise (ie, beta blockers and non-dihydropyridine calcium channel blockers)

\section{During Recovery}

- Arrythmias: Frequent ventricular ectopy (defined as $\geq 7$ VPBs per minute, ventricular bigeminy or trigeminy, or more marked ventricular arrhythmia)

- ECG changes $: \geq 1 \mathrm{~mm}$ ST segment depression

- Heart Rate Recovery (HRR): Defined as difference in heart rate at peak exercise and at 1 minute after cessation of exercise. A HRR of 12 beats/min or less during an upright cool down period will be considered as positive test result.

\section{Exclusion Criteria}

1. All patients with contraindications to Exercise Testing which includes:

o Absolute

- Acute myocardial infarction (within $2 \mathrm{~d}$ )

- High-risk unstable angina

- Uncontrolled cardiac arrhythmias causing symptoms or hemodynamic compromise

- Symptomatic severe aortic stenosis

- Uncontrolled symptomatic heart failure

- Acute pulmonary embolus or pulmonary infarction

- Acute myocarditis or pericarditis

- Acute aortic dissection

o Relative

- Left main coronary stenosis

- Moderate stenotic valvular heart disease

- Electrolyte abnormalities

- Severe arterial hypertension (systolic blood pressure of $>200 \mathrm{~mm} \mathrm{Hg}$ and/or diastolic blood pressure of $>110 \mathrm{~mm} \mathrm{Hg}$.)

- Tachyarrhythmias or bradyarrhythmias

- Hypertrophic cardiomyopathy and other forms of outflow tract obstruction

- Mental or physical impairment leading to inability to exercise adequately

- High-degree atrioventricular block

2. All patients unable to perform exercise tread mill test.

3. All patients with known CAD

4. All Patients with the following baseline ECG abnormalities:

o Pre-excitation (Wolff-Parkinson-White) syndrome

o Electronically paced ventricular rhythm

o Greater than $1 \mathrm{~mm}$ of resting ST depression

o Complete left bundle-branch block

\section{Study Assessments}

All of patients with stress test positive went through a detail interview regarding personal details like name, age, gender, profession along with an appropriate medical history of chest pain. History of Coronary artery disease risk factor: Hypertension, Dyslipidemia, Cigarette Smoking, Family history of CAD, diabetes and sedentary life style. Each patient had his/ her height and weight measured for calculation of BMI.

\section{Ascertainment of Coronary artery disease}

All patients with test positive results underwent coronary angiographic in Manmohan Cardiothoracic Vascular and Transplant Center CATH Lab by cardiology faculty member or cardiology resident. Vascular access for coronary angiography was done either by femoral or radial artery approach. Interpretation of coronary angiogram for $\mathrm{CAD}$ and coronary lesion quantification was done in at least two orthogonal views.

The criterion for Significant Coronary artery disease (CAD) was defined as $>50 \%$ stenosis of the LMS, $>70 \%$ stenosis in a major coronary vessel $\{$ Left Anterior Descending (LAD), Left Circumflex (LCX)or Right Coronary Artery( RCA)\} in coronary angiography ; Borderline significant as $50-70 \%$ Stenosis and minor or insignificant $\mathrm{CAD}$ as $<50 \%$ stenosis. Patients with significant coronary lesions were further classified depending on the number of major coronary vessel lesion as having single vessel disease (SVD), Double Vessel Disease (DVD) or Triple Vessel Disease (TVD).

\section{Statistical Analysis}

We estimated that a sample size of 132 patients would be required to obtain a two-sided $95 \%$ confidence interval for an estimated population of approximately 200 patients undergoing coronary angiography after stress test.

The prevalence of CAD and the associated $95 \%$ confidence interval were calculated for the entire group of patients and for relevant subgroups. To compare the baseline characteristics between patients with and those without CAD, we used the chi-square test for categorical variables and Student's t-test for continuous variables. Odds ratios with $95 \%$ confidence intervals were calculated with the use of logistic regression. The $95 \%$ confidence intervals and $\mathrm{P}$ values were calculated according to the normal approximation of the binomial distribution. No adjustments were made for multiple testing. All calculations were performed with the use of SPSS software, version 18.0 (SPSS).

\section{Results}

\section{Patient Characteristics}

A total of 303 patients (mean age $52.8 \pm 9.7 \mathrm{yrs}$ ) were included during a study period of approximately 1 and $1 / 2$ yrs (Oct 2015 to Dec 2016) among whom male were of average $53.6 \pm 10.5 \mathrm{yrs}$ and female were of $51.7 \pm 8.6 \mathrm{yrs}(\mathrm{p}$ value-0.098). It was observed that more female patients complained of chest pain than males \{male $39.27 \%(n-119)$ vs $60.72 \%(n-184)\}$.

Among all the patients who underwent TMT greatest number of the patient were hypertensive $\{47.85 \%(n-145)$ and least number of patients were smoker $\{5.94 \%(n-18)\}$ with patients having at least one risk factor being $65.96 \%(n-188)$ and those with two or more risk factor being $34.03 \%$ (n-97) which was significantly higher in male than female(p - $0.196, \mathrm{p}-0.036$ respectively). Gender based analysis also revealed that most of risk factors were similar except smoking which was higher in female than male $(66.66 \%$ vs $33.33 \%$ respectively, p value- 0.595$)$ and more female obese than male $(66.66 \%$ vs $33.33 \%$ respectively, $\mathrm{p}$ value- 0.059$)$ as shown in Table 1 . 


\begin{tabular}{|c|c|c|c|}
\hline \multirow{2}{*}{ Characteristics } & \multicolumn{2}{|c|}{ Total Patients (N-303) } & \multirow{2}{*}{ P valus } \\
\hline & Male (n-119) & Female (n-184) & \\
\hline Age of patient(yrs) & $53.6 \pm 10.5 \mathrm{yrs}$ & $51.7 \pm 8.6 \mathrm{yrs}$ & 0.098 \\
\hline Complaint of chest pain & $39.27 \%(n-119)$ & $60.72 \%(n-184)$ & 0.023 \\
\hline \multicolumn{4}{|l|}{ Risk Factor for CAD distribution } \\
\hline Hypertensive (n-145) & $52.9 \%(\mathrm{n}-63)$ & $44.6 \%(n-82)$ & 0.154 \\
\hline Diabetics (n-32) & $59.37 \%(\mathrm{n}-19)$ & $40.62 \%(n-13)$ & 0.661 \\
\hline Smoker(n-18) & $33.33 \%(n-6)$ & $66.66 \%(n-12)$ & 0.595 \\
\hline \multicolumn{4}{|l|}{ Body Mass Index(BMI) } \\
\hline Normal BMI(n-192) $(18.5-22.9$ kg/m2) & $35.41 \%(n-68)$ & $64.58 \%(n-124)$ & 0.016 \\
\hline Overweight (n-63) (BMI-23.0-27.5 kg/m2 ) & $66.66 \%(n-42)$ & $58.33 \%(\mathrm{n}-21)$ & 0.782 \\
\hline Obese(n-27) (BMI- $\geq 27.5 \mathrm{~kg} / \mathrm{m} 2)$ & $33.33 \%(n-9)$ & $66.66 \%(n-18)$ & 0.059 \\
\hline$\geq 2$ CAD risk factors $(n-95)$ & $65.26 \%(n-62)$ & $34.73 \%(n-33)$ & 0.036 \\
\hline At least 1 CAD risk Factor (n-205) & $66.82 \%(n-137)$ & $33.17 \%(n-68)$ & 0.196 \\
\hline
\end{tabular}

Coronary angiography of all the patients revealed normal coronaries in $54.0 \%(\mathrm{n}-114)$, minor CAD in $13.7 \%(\mathrm{n}-29)$ and significant lesion in $32.2 \%$ (n-68) (Fig1.)

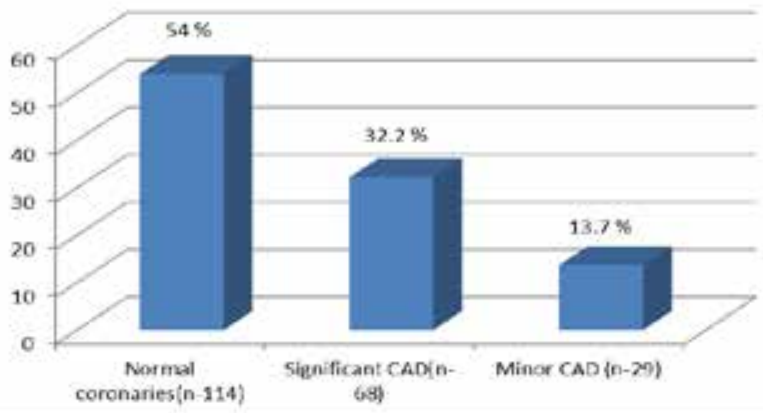

Fig 1. Percentage based on coronary angiographic finding

Among those with diagnosed CAD $22.7 \%(n-48)$ had single vessel disease (SVD), $13.7 \%(n-29)$ had double vessel disease (DVD), $6.6 \%(\mathrm{n}-14)$ had triple vessel disease (TVD) (Fig2.).

\section{Coronary Artery Disease}

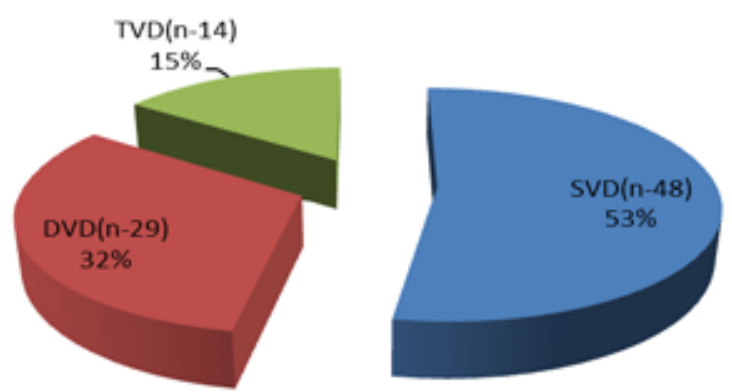

Fig 2. Distribution of coronary artery disease

The sub group analysis based on risk factor and CAD showed CAD was highest among diabetics $57.7 \%$, Odds ratio $1.72(95$ $\%$ CI:0.92 to 3.20 ), p value- 0.08 ) followed by smokers ( $55 \%$,Odds ratio 1.52 (95\% CI:0.59 to3.93), p value -0.38) compared with all the patient groups though not statistically significant.

\begin{tabular}{|c|c|c|c|c|c|}
\hline \multirow{2}{*}{$\begin{array}{l}\text { CAD Risk } \\
\text { Factors }\end{array}$} & \multicolumn{3}{|c|}{ CAG finding } & \multirow{2}{*}{$\begin{array}{l}\text { Odds Ratio } \\
(95 \% \mathrm{CI})\end{array}$} & \multirow[b]{2}{*}{ P Value } \\
\hline & $\begin{array}{c}\text { Normal Coronaries } \\
(\mathrm{n}-173)\end{array}$ & $\begin{array}{c}\text { Minor CAD } \\
(\mathrm{n}-41)\end{array}$ & $\begin{array}{c}\text { Significant CAD } \\
(\mathrm{n}-89)\end{array}$ & & \\
\hline HTN & $45.66 \%(n-79)$ & $51.21 \%(\mathrm{n}-21)$ & $50.56 \%(\mathrm{n}-45)$ & 1.00 (95\% CI:0.68 to 1.48$)$ & 0.97 \\
\hline $\mathrm{DM}$ & $10.98 \%(n-19)$ & $19.51 \%(n-8)$ & $20.22 \%(n-18)$ & 1.72 (95 \% CI:0.92 to3.20) & 0.08 \\
\hline Smoker & $(n-8)$ & $12.19 \%(\mathrm{n}-5)$ & $5.61 \%(n-5)$ & 1.5259 (95 \% CI:0.59 to3.93) & 0.38 \\
\hline \multicolumn{6}{|l|}{ BMI } \\
\hline Normal & $65.31 \%(n-113)$ & $73.17 \%(n-30)$ & $55.05 \%(n-49)$ & 0.75 (95 \% CI:0.52 to 1.08$)$ & 0.13 \\
\hline Overweight & $26 \%(n-45)$ & $26.82 \%(n-11)$ & $31.46 \%(n-28)$ & 1.05 (95 \% CI:0.65 to 1.68$)$ & 0.83 \\
\hline Obese & $8.6 \%(n-15)$ & 0 & $13.48 \%(n-12)$ & 1.01 (95 \% CI:0.46 to 2.21$)$ & 0.97 \\
\hline
\end{tabular}


Further analysis of patients comparing the risk of having $\mathrm{CAD}$ two or more CAD risk factors to at least one CAD risk factor showed significantly higher chance among patient with $\geq 2$ CAD risk factor (OR: 8.10,95 \% CI:4.96 to $13.24, \mathrm{P}<0.0001$ ).

Gender distribution of CAD showed that males with stress test positive had significantly higher chances of having CAD than females ( $53 \%$ vs $35 \%$ respectively) with odds ratio(OR) : $2.08,95$ \% CI: 1.30 to 3.32 ,p value- 0.002 ).

\section{Discussion:}

Graded exercise tread mill test is widely used clinically to assess the ability of an individual to safely tolerate increased physical activity while ECG, hemodynamic, and symptomatic responses are monitored for the development of myocardial ischemia, electrical instability, or other exertion-related abnormalities. Also exercise ECG remains an inexpensive test that has been well validated in the general population, and it can be used as the first diagnostic test for patients with an intermediate risk of having CAD.

Given the differences in presentation of CAD within the diabetic population and particularly the higher incidence of silent myocardial ischemia, exercise ECG testing has been used to assess asymptomatic diabetic patients for CAD. Koistinen et $\mathrm{al}^{5}$ studied 136 asymptomatic diabetic patients who underwent exercise ECG testing and was abnormal in $14 \%$ of these asymptomatic patients, with a positive predictive value of $94 \%$. Similar to the study sub group analysis based on of risk factor and CAD showed that $\mathrm{CAD}$ was highest among diabetics $(57.7 \%$, Odds ratio 1.72 (95\% CI:0.92 to3.20) ),p value-0.08) though not statistically significant which probably could be due to small sample size of the study group.

Bacci et $\mathrm{al}^{6}$ evaluated 206 consecutive higher-risk, asymptomatic, T2DM patients with peripheral arterial disease (PAD) and at least 2 cardiovascular risk factors (CRFs); 19\% had an abnormal test of those $29 \%$ had significant CAD. The positive predictive accuracy of the exercise ECG was 79\%. During the study comparing the risk of having CAD two or more CAD risk factors to at least one CAD risk factor showed significantly higher chance among patient with $\geq 2 \mathrm{CAD}$ risk factor (OR: 8.10,95\% CI:4.96 to $13.24, \mathrm{P}<0.0001$ ) which is comparable .

Nayak K C et al ${ }^{7}$ performed treadmill exercise test in 50 chronic smokers and 50 non-smokers (93 males and 7 females) who were not having any clinical or electrocardiographic manifestation of ischemic heart disease. The test was positive in $18 \%$ chronic smokers and $4 \%$ in non-smokers, the chances of positivity of stress test was 4-5 times greater in chronic smokers than in non-smokers. In study too smokers had higher chance of having CAD (55\%, Odds ratio 1.52 (95\% CI: 0.59 to 3.93 ), $\mathrm{p}$ value -0.38) compared with other patient groups though not statistically significant.

Miller TD et $\mathrm{al}^{7}$ directly compared the sensitivity and specificity of treadmill testing in 3,213 women vs. 5,458 men using myocardial perfusion as the reference standard. In the smaller subset of the study patients referred for coronary angiography (205 women, $838 \mathrm{men}$ ), the false-positive rate was again higher in women ( $13 \%$ vs. $7 \%, \mathrm{p}=0.003)$, but neither specificity $(69 \%$ vs. $74 \%, p=N S)$ nor accuracy $(60 \%$ vs. $66 \%, p=$ NS) was different between the sexes. Gender difference of CAD also seen in this study showed that males with stress test positive had significantly higher chances of having CAD than females ( $53 \%$ vs $35 \%$ respectively) with odds ratio(OR) : $2.08,95 \% \mathrm{CI}$ : 1.30 to 3.32 ,p value- 0.002 ).

In this study exercise test has proved to be important to detect early CAD in patient with stable ischemic heart disease but significantly more those of male gender, diabetics \& smoker or combination of two or more CAD risk factors compared to those of female in whom chances of having a false positive test positive is much higher. But the study has been limited by small sample size among subgroups of patients which may be the reason not finding a significant relation between the groups.

\section{Conclusion:}

The study shows that the pretest probability of detection of stable CAD with treadmill stress-test is highest in males with two or more $\mathrm{CAD}$ risk factor especially diabetes and smoking compared to the female counterparts with similar factors.

\section{References:}

1. Armin Arbab-Zadeh. Stress testing and non-invasive coronary angiography in patients with suspected coronary artery disease: time for a new paradigm.Heart Int. 2012; 7(1): e2.

2. Amany H Ahmed, KJ Shankar, Hossein Eftekhari, et al Silent myocardial ischemia: Current perspectives and future directions. Exp Clin Cardiol. 2007; 12(4): 189-196.

3. Gibbons RJ, Balady GJ, Bricker JT, et al. ACC/AHA 2002 guideline update for exercise testing: a report of the American College of Cardiology/American Heart Association Task Force on Practice Guidelines (Committee on Exercise Testing). 2002. American College of Cardiology Web site.

4. Abdou Elhendy, Ron T van Domburg, Jeroen J Bax. The significance of stress-induced ST segment depression in patients with inferior $\mathrm{Q}$ wave myocardial infarction. JACC. 1999;33(7):1909-1915

5. Koistinen M, Huikuir J, Pirttiaho H,et al . Evaluation of exercise electrocardiography and thallium tomographic imaging in detecting asymptomatic coronary artery disease in diabetic patients. Br Heart J. 1990; 63:7-11

6. Bacci S, Villela M, Villela A, et al. Screening for silent myocardial ischaemia in type 2 diabetic patients with additional atherogenic risk factors: applicability and accuracy of the exercise stress test. Eur J Endocrinol. 2002; 147: 649-654

7. Nayak KC, Gett SS, Sharda DP,et al . Treadmill exercise testing in asymptomatic chronic smokers to detect latent coronary heart disease. Indian Heart J. 1989; 41(1):62-5.

8. Miller TD, Hodge DO, Christian TF, et al. Effects of adjustment for referral bias on the sensitivity and specificity of single photon emission computed tomography for the diagnosis of coronary artery disease. Am J Med. 2002; 112(4):290-7.
Cite this article as: Amit Shrestha ,Sanjeev Thapa, Sheelendra Shakya,et al. Coronary angiography finding of exercise tread mill test positive patients in a tertiary care center.Nepalese Heart Journal 2017; 14(1): 17-20 